\title{
Fabrication and Testing of Ultra-Long Life Anode Material Using PEDOT-PSS/ Graphene Nanoplatelet Composite for Flexible Li-ion Batteries
}

\author{
Syed Khasim ${ }^{1,2,3, *}$, Nacer Badi ${ }^{1,2}$, Apsar Pasha ${ }^{4}$, S.A. Al-Ghamdi ${ }^{1,2}$, N. Dhananjaya ${ }^{5}$, S. Pratibha ${ }^{5}$ \\ ${ }^{1}$ Department of Physics, Faculty of Science, University of Tabuk, Tabuk-71491, Kingdom of Saudi \\ Arabia \\ ${ }^{2}$ Renewable Energy Laboratory, Nanotechnology Research Unit, Faculty of Science, University of \\ Tabuk, Tabuk-71491, Kingdom of Saudi Arabia \\ ${ }^{3}$ Department of Physics, PES University-Bangalore South Campus, Bangalore-560100, India \\ ${ }^{4}$ Department of Physics, Ghousia College of Engineering, Ramanagaram, Karnataka 562159, India \\ ${ }^{5}$ Centre for Advanced Materials Research Lab, Department of Physics, BMS Institute of Technology \\ and Management, Bangalore, Karnataka 560064, India \\ *E-mail: syed.pes@ gmail.com
}

doi: $10.20964 / 2021.02 .20$

Received: 11 October 2020 / Accepted: 1 December 2020 / Published: 31 December 2020

In this work, we report the fabrication of a robust, flexible and free-standing composite film electrode for Li-Ion batteries (LIBs) using a simple bar coating technique. The electrode consists of a conductive composite matrix of poly(3,4-ethylene-dioxythiophene)-poly(styrene sulfonate) (PEDOT-PSS): poly(acrylic acid) (PAA) loaded with Graphene nanoplatelets (GNPs). The successful coating of PEDOT-PSS: PAA on GNPs reduces the surface resistance and protects the electron channels on the GNPs surface from the pulverization effect during charging \& discharging cycles. This PEDOT-PSS: PAA: GNPs composite electrode shows excellent cyclability with an initial reversible capacity of 1325 $\mathrm{mAhg}^{-1}$ at a current density of $200 \mathrm{mAg}^{-1}$. The electrode also displays excellent capacity retention of $\sim 83 \%$ with a coulombic efficiency of $99 \%$ after 1000 cycles. This composite structure with a porous network induces physical strains in the structure during the Lithium (Li) intake- release process, thereby increasing the interfacial contact area with organic electrolyte. Consequently, the PEDOT-PSS: PAA: GNPs composite electrodes demonstrate improved capability without capacity fading. The composite film shows an improved Young's modulus of $2.4 \pm 0.18 \mathrm{GPa}$ to $4.6 \pm 0.18 \mathrm{GPa}$ and Tensile strength of $55 \pm 2.5 \mathrm{MPa}$ to $78 \pm 2.5 \mathrm{MPa}$ in comparison to pure PEDOT-PSS films. Benefiting from excellent conductivity, superior electrochemical performances, and mechanical flexibility, these hybrid systems could be potential materials towards the fabrication of flexible LIBs in wearable electronics.

Keywords: PEDOT-PSS, Graphene, Li-Ion, Anode, Flexible Batteries, 


\section{FULL TEXT}

(C) 2021 The Authors. Published by ESG (www.electrochemsci.org). This article is an open access article distributed under the terms and conditions of the Creative Commons Attribution license (http://creativecommons.org/licenses/by/4.0/). 